De la Barra y cols.

Rev. Chll. Pediatr. 66 (2); 112-117, 1995

\title{
Pesquisa de maltrato infantil y juvenil
}

\author{
Flora de la Barra M.'; Patricio Alvarez P. ${ }^{\text {; }}$ Virginia Toledo D. \\ Jorge Rodriguez T. ${ }^{2}$; Luis Caris N.'
}

\section{Inquiry on abuse in children and adolescent at a suburban county of Santiago, Chile}

\begin{abstract}
A method for cetection and registration of child abuse [demonsirated or suspecied] was experimentally applied of a suburban community of Santiago Chile as a contribution to the understanding of the problem in the country and to future development of a national case register. The questionnaire was applied by Irained personnel of health, education and justice institutions lo cases detected at heath centers, schools, police stations and justice courts ond to a paired control sample of unrelated coses from the some inslitutions. Children's characteristics, type of abuse and brological, social and family risk factors were recorded. Characteristics of 145 cases and 125 controls were compared, as well as subtypes of abuse, and different maternal ages. Significant differences were respectively recorded in the frequency of family members with mentral diseases (23.8\% vs. $11.2 \%$ ) that lived in the same household as the abused case $167.6 \%$ vs. $14.3 \%$; absence of alcohol comsumplion, (21\% vs. $44 \%$ ), problem alcohol drinkers $\{23.8 \%$ vs. $7.2 \%$, and illicit drug consumtion \{15.5\% vs. $7.2 \%$ \} among family members; behaviour problems of the child [54\% ante $16,8 \%]$; use of social aid programmes $[26.9 \%$ vs. $12,8 \%$ ]; attendance to public townhall managed

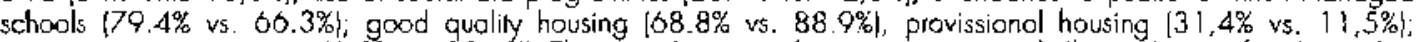
participation in communily $10.2 \%$ vs. $13.6 \%$. These results remain however provissional till complerion of wider studies with more refined instruments.
\end{abstract}

(Koy words: tantered child syndrome, child abuse.)

El maltrato a niños y adolescentes es objeto de creciente preocupación en la comunidad y en las diversas instituciones que atienden niños y adolescentes en Chile, pero la consulta espontánea por este motivo es baja, su detección y, en consecuencia, su denuncia, notificación y registro son insuficientes, surgiendo la necesidad de estimar su magnitud y las variables asociadas para desarrollar acciones y programas específicos de prevención, control y tratamiento del problema $^{1-5}$. Las acciones llevadas a cabo hasta ahora incluyen la promulgación de leyes que facilitan la protección de las victimas y la rehabilitación de las familias y la iniciación de pro-

1. Facultad de Medicina. Universidad de Chile. Departamentos de Psiquiatría y Salud Mental. Campus Oriente. Salud Pública. Campus Norte y Sur.

Servicio de Salud Metropolitado Suroriente.

2. Estadístico. Profesor Escueja de Salud Pública. Facultad de Medicina, Campus Sur.

Financiado por Ministerio de Planificación, UNICEF, Vicaría de Pastoral Social. gramas locales en dieciocho servicios de salud del país, apoyados por normativas ministeriales $s^{2,4-10}$.

El estudio que se presenta a continuación se realizó con los propósitos de probar, entre varios posibles ${ }^{1-19}$, un método de detección y registro de maltrato en personas menores de 18 años, y analizar algunas variables sociales, biológicas y demográficas de riesgo, basándose en los casos denunciados o detectados por sospecha clínica fundada en servicios de salud, instituciones educacionales o judiciales en una comuna de Santiago.

\section{Material y Método}

Se realizó un estudio de casos y controles. Los casos fueron los nitios ea que se identific 6 o sospechó fundadamente el maltrato, definido como daño o deterioro provocado por actos voluntarios o marcada desantención por parte de los padres o cuidadores, cuyas formas fueron descritas detalladamente en el instrumento de registro. Los controles fueron nifios pareados por edad, seleccionados 
entre los consultantes de la misma institución mediante el procedimiento de elegir de la nómina correspondiente al siguiente consultante o denuncia. Para evitar doble registro de los casos detectados en distintos establecimientos se aplicó a cada uno de ellos una clave de identificación constituida por la fecha de nacimiento y las iniciales de su nombre $y$ apellido. Se aseguró por todos los medios la confidencialidad de los datos obtenidos. Les casos detectados fueron derivados para tratamiento de acuerdo al programa vigente para maltrato infantil en el servicio de salud correspondiente a la localidad.

En el período de observación se incluyeron todos los casos registrados durante esta etapa en las instituciones seleccionadas, clasificadas en un sector judicial (grupo l: juzgados de menores, policia local y del crimen: comisarías policiales uniformada y civil; Instituto Médico Legal: Servicio Nacional de Menores, Servicio Nacional de la Mujer). otro de salud (grupo 2: servicio de argencia, Hospital Sótero del Rilo, consultorios de atención primaria y centro comunitario de salud mental familiar) y atro de educación (grupo 3: escuelas, jardines infantiles y salas cunas).

El tamaño de la muestra fue estimado en 350 niños ( 175 casos e igual número de controles) y el de las pérdidas en $25 \%$. Una vez jdentificado un caso por el profesional tratante en las instituciones de salud, un entrevistador capacitaóo registró en el formulario los antecedentes obtenidos del acompañante adulto del niño y seleccionó al control repitiendo con él el procedimiento de registro en la misma forma. En las escuelas, liceos y jardines infantiles los profesores capacitados instruyeron a sus colegas jefes de curso para identificar a alumnos bajo sospechn de ser maltratados y llenaron los formularios, seleccionando como control al siguiente alumno en la lista del curso. Cuando faltaron dalos, ciló al apoderado. En comisarías policiales, Instituto Mćdico Legal y juzgados el registro y la evaluación se efectuaron en dos etapas: una visita a la institucion, revision de expedientes, libros de denuncias y peritajes para identificar casos y controles $y$ Juego entrevista a un familiar adulto en domicilio del niffo.

El instrumento de registro consta de cinco secciones (su versión completa está disponible en la dirección del autor) constituidas por la identificación del nif̂́o y la persona que lo acompafia, domicilio, comuna, tipo de vivienda, colegio y consultorio: antecedentes familiares, rol de cada miembro respecto de la situación de maltrato, sospecha o caso confinmado de maltrato infantil, factores de riesgo familiares; características del niño maltratado y factores de riesgo personales (embarazo. parto, enfermedades crónicas. problemas conductuales); tipo y severidad del maltrato y, por último, una para describic la situacion de maltrato y las lesiones observadas. El cuestionario fue conocido, discutido y modificado por un equipo asesor del Ministerio de Planificación, quedando finalmente integrado por 248 variables. Se decidjó utilizar el formulario completo en todas las instituciones para evaluar la calidad de la información obtenida en cada una de las secciones en los distintos tipos de institución.

En octubre de 1993 se efectuó la coordinación de terreno, realizando entrevistas con jefes, directores y otros profesionales de todas las instituciones involucradas en el proyecto $y$, un mes después, se hicieron tres talleres de capacitación para profesores, educadoras de párvulos, pro- fesionales de la salud y los estudiantes de ciencias médicas que realizarían las visitas domiciliarias en casos detectados en comisarias y juzgados. Se nombró un profesional coordinador por cada lugar de trabajo encargado de centralizar y revisar encuestas y se tefinió el flujograma para el perfoto de observación.

El perfodo de observación del estudio de campo fue de cinco semanas en educación, cuatro en salud y tres en justicia, comenzando en noviembre y diciembre 1993. Las encuestas cumplidas fueron revisadas centralmente por supervisores, codificadas e ingresadas a computación. Para el análisis de la información se utilizo el paquete estadistico EPINFO. La información obtenida fue evaluada mediante pruebas estadísticas de Mantel-Hanzel, riesgos relativos $y$ análisis de varianza.

El número de casos susceptibles de ser pesquisados en el sector justicia fue menor que lo estimado, posiblemente debido a dificulıades de acceso a los lugares donde se efeclúan las denuncias y ausencia de registros específicos del maltrato. No había casos registrados en el centro comuni1ario de salud mental familiar, la oficina local de servicio nacional de la mojer y el consultorio psiquiátrico, porque no atienden niños. En este último estrableciraiento se realizó la coordinación, capacitación y recolección centralizada de encuestas.

Las dificultades para llenar el instrumento de detección fueron: en salud, altos porcentajes de falta de respuesta sobre el tipo de parentesco con la familia (biologico o adoptado); en educacion, defectos en el registro de enfermedad mental, consumo de drogas en la familia y antecedentes de hospitalizacion de] niño y, en los tres sectores, deficiente descripción de las lesiones. El registro de trastomos conductuales en los niños maltratados fue notablemente bueno.

\section{Resultados}

El conjunto de encuestados (casos y controles) estuvo constituido por igual número de hombres y de mujeres, cuya edad promedio fue de 7,6 años, $60 \%$ cursaban educación básica, los restantes educación media, 3,7\% eran hijos adoptados. Setenta y tres y medio por ciento de los niños asistían a colegios municipales, $13 \%$ a escuelas privadas subvencionadas, $3,2 \%$ particulares y $10,3 \%$ otros. En $85,9 \%$ de los casos la vivienda era sólida, $28,6 \%$ la compartían. El tamaño promedio de las familias era de 5,2 personas, el número de camas por familia 3,7 . En la red comunitaria participaban $47,6 \%$ de los encuestados, $89,9 \%$ utilizaban servicios públi$\cos$ y $20,4 \%$ el servicio social.

En $17,9 \%$ de los encuestados había algún familiar con enfermedad mental, $52,1 \%$ de los cuales vivían con el niño. Se declaró nunca consumir alcohol en $31,7 \%$, bebedores problema en $16 \%$ y consumir otras drogas en $11,6 \%$ de las 
familias. Se registraron antecedentes de morbilidad del embarazo en $12.5 \%$, el parto en $21,9 \%$, enfermedades crónicas del niño en $15,4 \%$, discapacidad del niño $25 \%$ y antecedentes de hospitalización del niño en $14,8 \%$ de los casos. En $36,8 \%$ de los niños se describieron problemas conductuales, de los cuales $73.4 \%$ tenían conductas disruptivas y $8,1 \%$ conductas en déficit. Hubo diferencias significativas en la detección de problemas conductuales de los niños en los distintos sectores. La razon de disparidad fue de $2,34 \%$ en salud, $37,5 \%$ en educación y $5 \%$ en los juzgados. Se consignaron 201 personas como sospechosas de causar algún tipo de maltrato en los 145 casos identificados, 104 de aquéllas eran mujeres. La edad promedio de los presuntos autores era 34 años.

No se observaron diferencias significativas entre casos de maltrato demostrado o probable y controles en edad, sexo, escolaridad, calidad de hijo biologico o adoptado, independencia y tamaño del grupo familiar, número de camas, antecedentes de morbilidad del embarazo y cl parto, enfermedades crónicas, discapacidad y antecedentes hospitalización del niño.

Fueron significativamente diferentes entre los casos de maltrato y los controles el antecedente de algún familiar con enfermedad mental $(23,8 \%$ ante $11,2 \%)$ que vivía con el niño $(67,6 \%$ ante $14,3 \%)$; ausencia de consumo de alcohol en la familia ( $21 \%$ ante $44 \%$ ); bebedores problema $(23,8 \%$ ante $7,2 \%)$; consumo de drogas en la familia $(15,5 \%$ ante $7,2 \%)$; problemas conductuales del niño ( $54 \%$ ante $16,8 \%$ ); utilización de servicio social $(26,9 \%$ ante $12,8 \%)$; asistencia a escuela municipal $(79,4 \%$ ante $66,3 \%)$; vivienda s6lida $(68,8 \%$ y $88,9 \%)$, transitoria $(31,4 \%$ y $11,5 \%)$; participación en juntas de vecinos $(6,2 \%$ ante $13,6 \%)$ y falta de participación en la red comunitaria $(60,1 \%$ y $43,5 \%)$ respectivamente. La frecuencia de respuestas "no sabe" fue significativamente mayor en casos que en controles.

En lo que respecta al tipo de maltrato en cađa niño, sólo $15,2 \%$ de los casos estaban sometidos a una sola forma de abuso, $29 \%$ a dos, otros $29 \%$ a tres tipos y $26,8 \%$ a cuatro o más. La frecuencia de las diferentes formas de maltrato fue, por separado, emocional y descuido emocional $67,7 \%$ (98 casos); maltrato físico $66,9 \%$ (97 casos); descuido físico: $50,3 \%$ (73 casos); descuido emocional: $12,4 \%$ (18 casos);
Tabla

Númera de casos y controles obtenido en el período de observación

\begin{tabular}{lccr}
\hline Institución & Casos & Controles & Total \\
\hline Grapo I & & & \\
Consultorios & 19 & 19 & 38 \\
Servicio de Urgencia & 48 & 50 & 98 \\
Subiotal salud & 67 & 69 & 136 \\
Grupo II & & & \\
Jardines infantiles & 12 & 7 & 19 \\
Escuelas y liceos & 60 & 45 & 105 \\
Subtotal educación & 72 & 52 & 124 \\
Grupo III & & & \\
Carabineros & & 4 & 8 \\
Juzgado de menores & 2 & & 10 \\
Subtotal judicial & 6 & 4 & 270 \\
\hline Total & 145 & 125 & \\
\hline
\end{tabular}

abuso sexual y otro tipo de maltrato: $8,3 \%(12$ casos).

Al cotejar cada tipo de maltrato con el conjunto de los casos, se encontraron asociaciones significativas entre: maltrato físico con ausencia de participación social y mayor proporción de familiares con bebedores problema y consumo de drogas; maltrato emocional con ausencia de participación comunitaria; abuso sexual con predominio de sexo femenino ( 11 de los 12 casos); enfermedad mental, bebedores problema y consumo de drogas en la familia; abandono físico con menor porcentaje viviendas sólidas y menor participación en organizaciones comunitarias.

La distribución de la edad de la madre al nacer el niño no mostró diferencias significativas entre casos y controles. Sin embargo, entre los hijos de madres menores de 20 años hubo. entre los maltratados, significativamente mayor proporción de familiares con enfermedades mentales (maltrato físico, abandono físico y emocional); bebedores problema (maltrato físico); consumo de drogas (maltrato físico, abandono físico y emocional); problemas conductuales del niño (maltrato físico, emocional, abandono físico y emocional) y menor porcentaje de casas sólidas (todos los tipos de maltrato).

Al comparar el subgrupo de hijos de madres adolescente con el total en cada tipo de maltrato, las variables de riesgo son drogadicción en 
familiar (maltrato emocional, abandono físico y emocional); falta de independencia del grupo familiar (maltrato físico, abandono físico y abandono emocional); ausencia de participación social (abandono físico y emocional).

\section{Comentario}

Los resultados expuestos provienen de un estudio piloto, por lo cual deben ser interpretados con la cautela inherente a esta etapa de la investiqación. Las diferencias consignadas entre grupos son estadísticamente significativas, sin embargo, no constituyen hallazgos definitivos. Más bien abren interrogantes a explorar y permiten formular hipótesis que deberán ser estudiadas en profundidad en una población representaljva nacional.

En la comparación entre casos y controles no aparece la mayor proporción de hombres sujetos a maltrato físico que señala la literatura sobre esta materia, para lo que cabe plantearse, como explicación, la posibilidad de sesgo de selección por un probable apareo involuntario por parte de los encuestadores.

La mayor proporción de asistentes a escuelas municipales entre los niños maltralados también debe ser estudiada con mayor detalle, ya que puede tratarse de una variable asociada de confusión, ya que si bien casos y controles provienen de una misma escuela, por el sistema de pareo, la diferencia podria estar dada por otra variable no controlada, como por ejemplo la situación social y económica.

Con respecto a las características del perpetrador, la proporcion levemente mayor de mujeres $(51,7 \%)$ no es comparable con otros estudios nacionales, ya que no incluyen todos los tipos de maltrato. La edad del agresor (adulto joven) coincide con todos los estudios conocidos.

La vivienda transitoria, ausencia de participación de la familia en la red comunitaria y mayor utilización de servicios sociales aparecen como factores de riesgo para el maltrato, lo que concuerda con estudios de otros países, que señalan distintos aspectos de la pobreza como productores de tensión familiar y aumento de] riesgo de maltrato ${ }^{21.22,24,26-30}$.

La existencia de familiares con enfermedad mental, bebedores problema y consumidores de drogas también han sido señalados como facto- res de riesgo para el maltrato en otras investigaciones $^{14,15,22,28,30}$. El consumo ilegal de drogas fue detectado solamente en las familias de niños abusados, en nuestro caso.

Los problemas conductuales del niño constituyen también un factor de riesgo para ser víctima de maltrato ${ }^{9}, 22,26-28$. Si bien las organizaciones de salud muestran mayor capacidad de detección, las de educación entregan mayor $r$ queza cualitativa en la información sobre la conducta de los niños. El número de conductas desadaptativas en los casos de maltrato detectados por los educadores, extremadamente más alto gue en los controles con respecto a los otros sectores, pudiese deberse a un particular esmero de parte de los maestros a seleccionar niños control lo más opuestos posible al caso, no sólo en el maltrato, sino también en las demás características, a pesar del criterio de selección.

La explicación a la mayor frecuencia de respuesta "no sê", consistentemente mayor en casos que controles, pudiera estar en un real desconocimiento por parte del informante, pero también en un intento de ocultar jnformación o en negligencia.

Uno de los objetivos del estudio fue pesquisar diversas formas simultáneas de maltrato en un mismo niño, lo que se registro en la mayoría de nuestros casos, en contraste con lo descrito en investigaciones nacionales precedentes, que sólo estudiaban y registraban formas aisladas de maltrato, generalmente castigo físico y abuso sexual ${ }^{5,9,15-18,30}$. El hecho que el maltrato y descuido emocional, formas tradicionalmente poco descritas, sean las categorías más frecuentes, apoya la importancia de las definiciones propuestas, la capacitación efectuada y la alta motivación de los profesionales encuestadores. El maltrato físico, que ocupa el tercer lugar, es casi tan frecuente como los anteriores, seguido del descuido físico. El abuso sexual aparece con pocos casos, debido a las dificultades específicas de acceso a información para este tipo de maltrato en los juzgados de menores, donde se informaron al menos veinte casos adicionales mientras uranscurría el estudio.

Las variables asociadas con cada tipo de maltrato, y los tipos de maltrato dentro de una población de casos, permiten identificar diferencias peculiares en la correlación con dichas variables, las que es preciso analizar en mayor número de casos en investigaciones posteriores. 
Los resultados descritos muesiran que es posible pesquisar casos en las instituciones de salud y educación e identificar las dificultades que deben resolverse para logrario en el sector judicial. El marco apropiado para la realización de un estudio de prevalencia nacional incluye la participación de todos los sectores involucrados. En cada uno de ellos debe adecuarse el instrumento de registro en forma específica, conservando un núcleo de información básica que permita comparar la obtenida. Los futuros estudios requerirán disponer de tiempo suficiente para cumplir todas las etapas (contacto con las instituciones, sensibilización de la población en estudio, formalización del trabajo a efectuar, capacitación de encuestadores y coordinadores locales, trabajo de campo, procesamiento y análisis de los datos).

Este y otros estudios contribuyen al desarrollo de un registro nacional centralizado, facilitando información actualizada sobre la prevalencia del problema, como también el acceso a los casos de maltrato y sospecha, con fines de prevención y rehabilitación.

\section{Resumen}

Como un aporte al conocimiento de la magnitud del problema del maltrato a niños y adolescentes en el país y a la organización de un sistema nacional de registro, se probó un método de detección de casos o sospecha fundada de maltrato en una comuna de Santiago. EJ cuestionario fue aplicado por personal capacitado en establecimientos de salud, educación y justicia. Se registraron las características de los niños, el tipo de maltrato y algunas variables de riesgo biológico, social y familiar. Se compararon las características de 145 casos y 125 controles, de subgrupos de maltrato y de madres adolescentes con otras edades, obteniendo diferencias significativas en las respectivas frecuencias de familiar con enfermedad mental $(23,8 \%$ ante $11,2 \%)$ que vivía con el niño $(67,6 \%$ ante $14,3 \%)$; ausencia de consumo de alcohol en la familia $(21 \%$ ante $44 \%)$, bebedores problema $(23,8 \%$ ante $7,2 \%$ ); consumo de drogas en la familia $(15,5 \%$ ante $7,2 \%)$; problemas conductuales del niño (54\% ante $16,8 \%$ ); utilización de servicio social $(26,9 \%$ ante $12,8 \%)$; asistencia a escuela municipal $(79,4 \%$ ante $66,3 \%)$; vivienda sólida
$(68,8 \%$ y $88,9 \%)$, transitoria $(31,4 \%$ y $11,5 \%)$; participación en juntas de vecinos $(6,2 \%$ ante $13,6 \%$ ) y falta de participación en la red comunitaria $(60,1 \%$ y $43,5 \%)$. Estos resultados iniciales deben ser ampliados y perfeccionados con estudios más amplios e instrumentos refinados.

(Palahras clave: maltrato infantil, síndrome de niño maltratado.)

\section{Agradecimientos}

A los profesionales y personal del servicio de urgencia del Hospital Dr. Sótero del Río, consultorios, escuelas. juzgados y comisarías donde se readizó esta investigación.

\section{Referencias}

1. Anónimo. [nforme final Comisión Interministerial para la Prevención de la Violencia Intrafamiliar. República de Chile. Enero 1993.

2. Nowa $F$, De la Barra $F$, Alvarez $P$, Gitchrist A, Alvarez $N$, Gamboa R: Maltrato infantil en Chile. Rev Chil Pediatr 1992; 63 (Supl. 2): 1-13.

3. Anónimo: Repúbtica de Chile: Metas y líneas de acción en favor de la infancia. Compromiso con los ninoos de Chile para la década. Santiago, Chile. 1992.

4. Anonimo: Sociedad Chilena de Pediatría. Universidad de Chile. Faculiad de Medicina. UNICEF. Ministerio de Salud. Protección de los nit̄os y atención integral del maltrato infantil, Chile. 1993.

5. Unicef: Analisis de situación. Menores en circunstancias especialmente difíciles. Santiago de Chile, mayo de 1991

6. República de Chile: Ministerio de Justicia. Introduce modificaciones a ta Ley $\mathrm{N}^{*} 16.618$ en matería de mal. trato a menores. 19-8-1994.

7. Republica de Chile: Ministerio de Justicia. Establece normas sobre procedimientos y sanciones relativos a los actos de violencia intrafamiliar. 19-8-1994.

8. República de Chile: Ministerio de Salud. Unidad de Salud Mental. Programas de Salud Mental Infantojuvenil. Nornias técnicas y programáticas para la prevencion y atención integral del maltrato infantil en los servicios de salud, Marzo 1994.

9. Alvarez P: Síndrome del niño Inaltratado. En Winter. A., Puentes R. eds. Medicina Infantil. 1' Edición. Santiago de Chile. Ed. Giorgio Emperiale. 1991: 1100. 1103.

10. Alvarez $P$, Olivarf $C$ : Terapia coactiva del maltrato infantil: Ia rehabilitacion fandiar en el contexto judicial. PSYKHE 1993; 2: 53-58.

11. National Center on Child Abuse and Neglect, Children's Bureau, Administration for Children, Youth and Families, Office of Human Development Services, U.S. Department of Health and Human Services. Study Findings. National Study of the Incidence and Severity of Child Abuse and Neglect Sept 1981. 
12. National Center on Child Abuse and Neglect, Children's Bureau. Administration for Children, Youth and Families, Office of Human Development Services, U.S. Department of Health and Human Services. "Study Findings. Study of Mational Incidence and Prevatence of Child Abuse and Neglect: 1988.

13. Wyatt $G$, Peters $S$ : Methodological considerations in research on the prevalence of child sexual abuse. Child Abuse \& Neglect 1986; 10: 241-251.

14. Zuravin S: The Ecology of Child Abuse and Neglect: Review of the Literature and presentation of data. Violence \& Victims, $1989 ; 4: N^{\circ} 2$

15. Florenzano $R$. Pino P. Xaplan M. Butrows J: Frecuencia de maltrato infantil y sexual en escolares de Santiago de Chile: Antecedentes familiares y consecuencias. Documento $N^{*} 56$ Adolescencia 8, 5-18, 1993. Depto. Psiquiatsia y Salud Mental. Facultad de Medicina Oriente. Universidad de Chile.

16. Vargas N, Lopez D. Perez P, Toro G. Zúniga P, Ciocca $P$ : El castigo f́́sico a los niños: opinión y conducca de los adultos. Rev Med Chil 1993: 121:567-573.

17. Vargas $N$, López $D$, Perez $P$, Zúniga $P$, Toro $G$. Ciocca P: Características del castigo físico intantil administrado por padres de tres colegios de Santiago. Rev Chil Pediatr 1993; 64: 333-336.

18. Zubarew $T$, Muddaleno $M$, Valenzuela $V$, et at: Sindrome del cin̄o maltratado en la Unidad de Adolescencia del Consultorio La Faena. Rev Chil Pediatr 1992; 63:71-72.

19. Finkelhor D, Hotoling G: Sexual abuse in the national incidence study of child abuse and neglect: an appraisal, Child abuse \& Neglect 1984;8: 23-33.
20. Greenwood $C$, Tangalo E, Maruta $T$ : Prevalence of sexual abuse, physical abuse and concurrent traumatic life events in a general medical population. Mayo Clin Proc 1990; 65: 1067-1071.

21. Inemational Pediatric Seminar: Child Abuse, Neglect and Violence. A Latin American Perspective. Santo Domingo, República Dominicana. Dic. 2-6. 1990. En Interoational Child Health; Vol 2, $\mathrm{N}^{\circ} 1, \mathrm{Ja}-$ nuary 1991. An Intemational Pediatric Association Publication in collaboration with UNICEF and WHO.

22. Leventhal J: Risk Factors for Child Abuse: Methodologic Standards in Case Control Studies. Pediatrics 1981; 68: 684-690

23. I Have there been changes in the epidemiology of sexual abuse in the 20th century? Pediatrics 1988; 82; $766-773$.

24. Skuse D: Enotional abuse and neglect. BMI 1989; 298: 1962-1964.

25. Wyatt $G$, Peters $S$ : Issues in the definition of sexual abuse in prevalence research. Child Abuse \& Neglect 1986: 10:231-240.

26. Child Abuse. Pediatric Clinics Agosto 1990.

27. Dumn Smith: Child abuse. Newsletter A.C.P.P. 1989; 11: 3 .

28. Starr H. Ed: Child Abuse Prediction. Policy implications. Ballinger Publishing Co. 1982; 105-134.

29. UNICEF. Consejo de Admınistracion. 1986. La Situation des Enfants Victims de Mauvais Traitements et Privés des Soins Vue dans un Perspective Mondiale.

30. Gilchrist A 1983: Sindrome del niño golpeado. Tesis de grado para optar al título de especialista en Psiquiatría Infantil. 